\title{
The Effective QR Code Development using VB.NET
}

\author{
Neeraj Bhargava \\ Dept. of Computer Sciences, \\ School of Engineering and \\ System Sciences, MDS \\ University, \\ Ajmer, India.
}

\author{
Ritu Bhargava \\ Department of MCA, \\ Govt. Women Engineering \\ College, \\ Ajmer, India
}

\author{
Manish Mathuria \\ Dept. of Computer \\ Engineering and Information \\ Technology, Govt. \\ Engineering College, \\ Ajmer, India
}

\author{
Khushboo Mantri \\ Dept. of Computer Science \\ of Engineering \& System \\ Sciences \\ MDS University, \\ Ajmer, India
}

\begin{abstract}
Product is not in range to the local public until they have not required information about the product. The QR (Quick Response) code provides a medium, so the consumer can qualified the product. QR code is very popular because of its capability of handle information with the resistant environment. But sometimes, QR code fails due to its manufacturing constraints and the limitation of the storing space. The objective of research is to analyze and conclude the feature characteristics of QR code with the development of effective QR code using VB. NET functionality. It considered that innovators are interest in the use of barcodes to encode more information per area unit than regular, black and-white barcodes. The QR code is nothing but an image that require a special digital QR Code Reader application. This research paper also discuss about the structure, symbology and properties of barcodes.
\end{abstract}

Keywords: QR code, VB.Net, QR Code Reader.

\section{INTRODUCTION}

QR codes are two-dimensional bar codes that can contain any alphanumeric text and often feature URLs that direct users to sites where they can learn about an object or place (a practice known as "mobile tagging"). Decoding software on tools such as android phones interprets the codes, which represent considerably more information than a one-dimensional code of similar size. The codes are increasingly found in places such as product labels, billboards, and buildings, inviting users (android mobile) to pull out their mobile phones and uncover the encoded information. Codes can provide tracking information for products in industry, routing data on a mailing label, or contact information on a business card. Small in size, the code pattern can be hidden or integrated into an esthetically attractive image in newspapers, student mark sheet, confidential paper, magazines, or clothing etc. [1].

QR Code (abbreviated from Quick Response Code) is the trademark for a type of matrix barcode (or two-dimensional code) first designed for the automotive industry. More recently, the system has become popular outside the industry due to its fast readability and large storage capacity compared to standard UPC barcodes. The code consists of black modules (square dots) arranged in a square pattern on a white background [2].

The information encoded can be made up of four standardized kinds ("modes") of data (numeric, alphanumeric, byte/binary, Kanji), or through supported extensions, virtually any kind of data. Unlike the old bar code that was designed to be mechanically scanned by a narrow beam of light, the QR code is detected as a 2-dimensional digital image by a semiconductor image sensor and is then digitally analyzed by a programmed processor. The processor locates the three distinctive squares at the corners of the image, and normalizes image size, orientation, and angle of viewing. The small dots are then converted to binary numbers and validity checked with an error-correcting code [2].

\section{QR CODEAPPLICATIONS}

QR codes are popular in Japan, where they are used for commercial tracking, logistics, inventory control, and advertising. Their popularity is climbing in Europe, the United
States, and Canada as people increasingly use mobile phones to access $3 \mathrm{G}$ networks. In England, a grant-funded venture led by Andy Rams den at Bath University investigates ways to use QR codes in academic settings. In physical learning spaces, QR codes might indicate what types of learning take placein each area or provide a link to schedulingsoftware that offers the opportunity to reserve a room [2].

QR codes might also be effective repositories of data in problem-solving. In early years QR code generally useful in industries for advertisement, packaging, etc. The dissemination of the smart phones has putted Barcode Reader in everyone's pocket. As a result, the $\mathrm{QR}$ code has become a focus of advertising strategy, since it provides quick and effortless access to the brand's website. QR Codes are now used over a much wider range of applications, including commercial tracking, entertainment and transport ticketing, product/loyalty marketing, e-learning using ICT, and in-store product labeling. It can also be used in storing personal information for use by government [3].

\section{THE BACKGROUND OF QR CODE DEVELOPMENT}

In 1970, IBM developed UPC symbols consisting of 13 digits of numbers to enable automatic input into computers. These UPC symbols are still widely used for Point-Of-Sale (POS) system. In 1974, Code 39 which can encode (symbolize) approx. 30 digits of alphanumeric characters was developed. Then in the early $1980 \mathrm{~s}$, multistage symbol codes whereapprox. 100 digits of characters can be stored such as Code $16 \mathrm{~K}$ and Code 49 were developed. As information rapidly developed in the recent years, requests had mounted for symbols which can store more information and represent languages other than English. To enable this, a symbol with even higher density than multistage symbols was required. As a result, QR Code, which can contain 7,000 digits of characters at maximum including Kanji Characters?(Chinese characters used in Japan) was developed in 1994.The history until realizing high-capacity and high-density symbols can be described as illustrated in Figure 1 when seeing them from the technology's aspect. Firstly, Interleaved 2 of 5 and Coda bar which can encode (symbolize) numbers were developed, 
followed by the development of Code 39 which can encode alphanumerical characters. Along withthe information developments, it had become necessary to have full ASCII encoded, and this resulted in the development of Code 128. Then, multistage symbols were developed where these linear symbols were arranged in several stages. Toyota Motor's Kanban Code is the world's first multistage symbol. As computers became popular, these codes developed into multirow symbols where multistage codes were extended and into matrix symbols where data were arranged in matrix. The printing areas for matrix symbols are the smallest among all, and is seen as highly prospective as the main symbol for the future.QR Code is a matrix symbol which has been developed as the one enabling all of high capacity PDF417, high density printing of data matrix, and high speed reading of maxi code based on the research made on their characteristics. Twodimensional symbols generally contain much more data amount when compared with linear symbols (approx.100 times more), and therefore require much longer data processing time and more complex process. Therefore, QR Code has had much consideration for its finder pattern to enable high-speed reading [4].

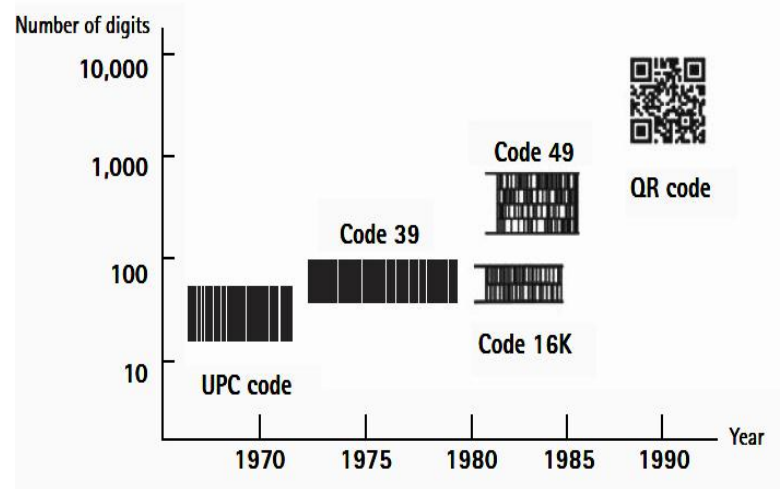

Figure 1: The History of Symbols

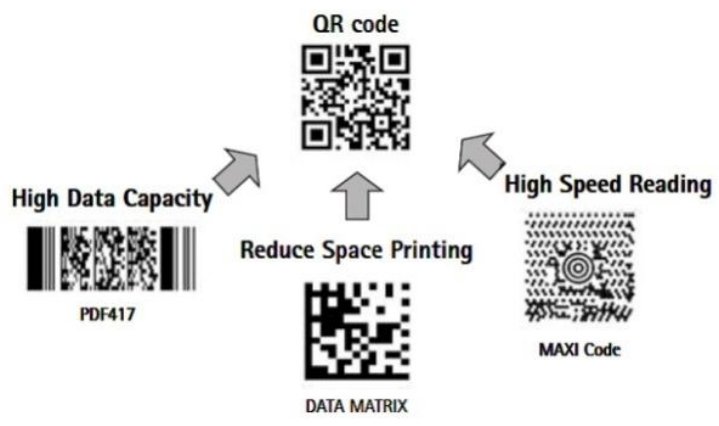

Figure 2: The Development of QR code

\section{CHARACTERISTICS OF THE QR CODE}

Additional to the characteristics for two-dimensional symbols such as large volume data $(7,089$ numerical characters at maximum), high-density recording (approx. 100 times higher in density than linear symbols), and high-speed reading, QR Code has other superiority in both performance and functionalities aspects.

\subsection{All-Direction $\left(360^{\circ}\right)$ High-Speed Reading}

Reading matrix symbols will be implemented by using a CCD sensor (area sensor). The data of the scan line captured by the sensor will be stored into the memory. Then, by using the software, the details will be analyzed, finder patterns identified, and the position/size/angle of the symbol detected, and the decoding process will be implemented.

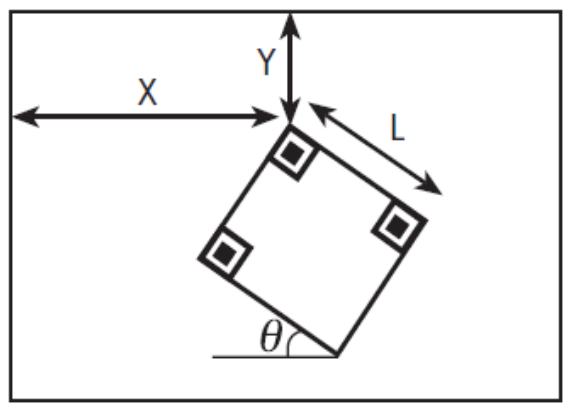

Figure 3: Resistant to Distorted Symbols

Symbols often get distorted when attached onto a curved surface or by the reader being tilted (angled between the CCD sensor face and the symbol face). To correct this distortion, QR Code has alignment patterns arranged with a regular interval within the range of the symbol. The variance between the Centre positions of the alignment pattern estimated from the outer shape of the symbol and the actual Centre position of the alignment pattern will be calculated to have the mappings (for identifying the Centre position of each cell) corrected. This will make the distorted linear/non-linear symbols readable.

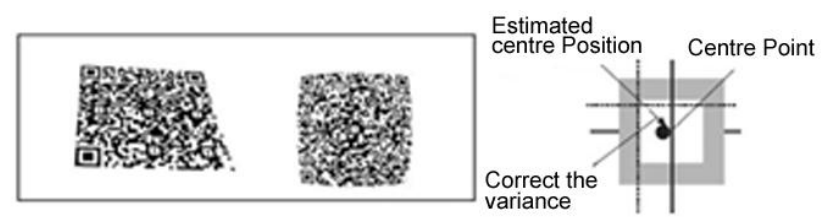

Figure 4: Correcting Distorted Symbol

\subsection{Data Restoration Functionality (Resistant to Smudged or Damaged Symbols)}

QR Code has four different error correction levels (7\%, 15\%, $25 \%$, and $30 \%$ per symbol area). The error correction functionality is implemented according to each of the smudge/damage, and is utilizing Reed-Solomon code which is highly resistant to burst errors. Reed-Solomon codes are arranged in the QR Code data area. By this error correction functionality, the codes can be read correctly even when they are smudged or damaged up until the error correction level.

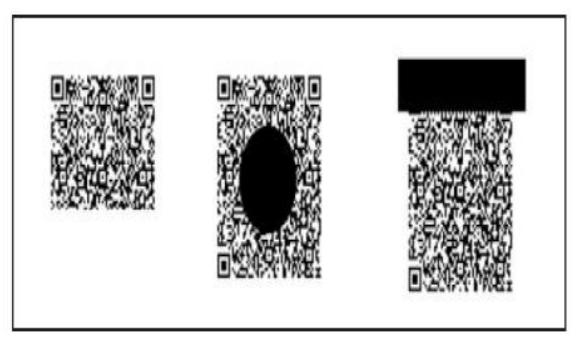

Figure5: Smudged/Damaged Symbols

\subsection{The Confidentiality of the Code}

By making the relationship between the character type and the stored data unique for a special usage, QR Code can be easily encrypted. Unless the conversion table between the character 
type and the stored data is deciphered, no one will be able to read the QR Code [7].

\section{THE QR CODE STRUCTURE}

The figure is version 1 (type 2) and the module is 21 X 21 cells, vertical 21 cells and horizontal 21 cells. This version is specified from 1 to 40 , increased by 4 cells per one version up. The maximum version is 40 and the size is $177 \mathrm{X} 177$ modules. Fig. 6 is a case of the QR code version 1 modules that are arranged in a grid pattern of black and white squares. In this QR code symbol [8][9][10], there are three position detection patterns (Finder patterns) in the upper left corner, bottom left and top right corner. Then the timing pattern is placed between every one of these position detection patterns. Additionally, alignment patterns are introduced in the version 7 or higher. Then Table I shows the main specifications of the QR code. There are four modes available,

(1) Number mode,

(2) Alphanumeric mode,

(3) 8 bit byte mode and

(4) Kanji and kana characters mode.

QR Code has finder patterns, alignment patterns, timing patterns, and a quiet zone.

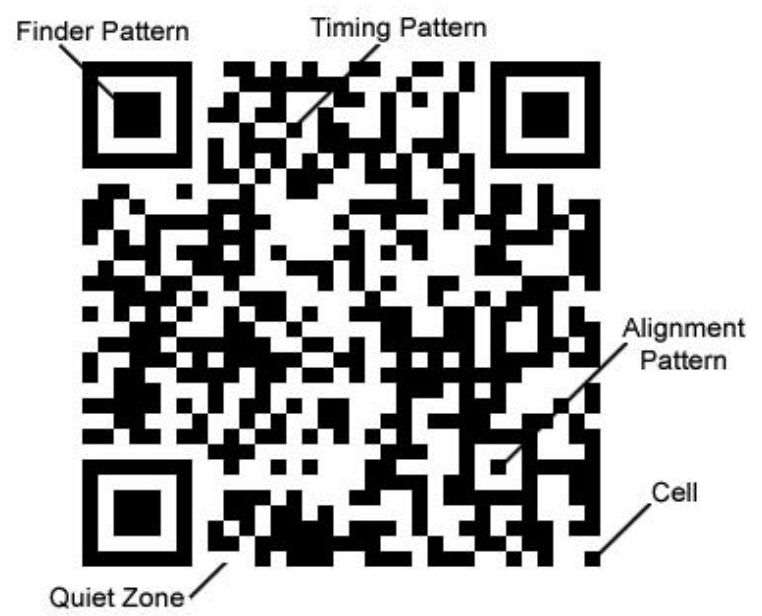

Figure6: QR Code Structure

\subsection{Finder Pattern}

A pattern for detecting the position of the QR Code. By arranging this pattern at the three corners of a symbol, the position, the size, and the angle of the symbol can be detected. This finder pattern consists of a structure which can be detected in all directions $\left(360^{\circ}\right)$.

\subsection{Alignment Pattern}

A pattern for correcting the distortion of the QR Code.It is highly effective for correcting nonlinear distortions. The central coordinate of the alignment pattern will be identified to correct the distortion of the symbol. For this purpose, a black isolated cell is placed in the alignment pattern to make it easier to detect the central coordinate of the alignment pattern.

\subsection{Timing Pattern}

A pattern for identifying the central coordinate of each cell in the QR Code with black and white patterns arranged alternately. It is used for correcting the central coordinate of the data cell when the symbol is distorted or when there is an error for the cell pitch. It is arranged in both vertical and horizontal directions.

\subsection{Quiet Zone}

A margin space necessary for reading the QR Code. This quiet zone makes it easier to have the symbol detected from among the image read by the CCD sensor. Four or more cells are necessary for the quiet zone.

\subsection{Data Area}

The QR Code data will be stored (encoded) into the data area. The grey part in Figure 11 represents the data area. The data will be encoded into the binary numbers of ' 0 ' and ' 1 ' based on the encoding rule. The binary numbers of ' 0 ' and ' 1 ' will be converted into black and white cells and then will be arranged. The data area will have Reed-Solomon codes incorporated for the stored data and the error correction functionality [4].

\section{THE SPECIFICATIONS OF THE QR CODE}

TABle1.The SPECIFICATIONS OF THE QR CODE ARE AS DESCRIBED

\begin{tabular}{|c|c|c|}
\hline Item & & pecifications \\
\hline \multirow{3}{*}{ Error } & Rs Code & Data \\
\hline & \multirow{2}{*}{ BCH Code } & Format Information \\
\hline & & Version Information \\
\hline \multirow{4}{*}{ Characters } & Number & $\begin{array}{c}10 \text { bit coding per } 3 \text { number } \\
\text { digits }\end{array}$ \\
\hline & Alphanumeric & 11 bit coding per 2 characters \\
\hline & 8 bit byte & 8 bit coding \\
\hline & Kanji & 13 bit coding per 2 characters \\
\hline \multirow{3}{*}{ Version } & 1 & $21 \times 21$ modules \\
\hline & 2 & $25 \times 25$ modules \\
\hline & 40 & $177 \times 177$ modules \\
\hline \multirow{4}{*}{$\begin{array}{c}\text { Error } \\
\text { Correcting } \\
\text { Level }\end{array}$} & $\mathrm{L}$ & About $7 \%$ \\
\hline & M & About $15 \%$ \\
\hline & Q & About $25 \%$ \\
\hline & $\mathrm{H}$ & About $30 \%$ \\
\hline \multirow{2}{*}{$\begin{array}{l}\text { Finder } \\
\text { Pattern }\end{array}$} & \multirow{2}{*}{$1: 1: 3: 1: 1$} & 3 co-centric squares \\
\hline & & $7 \times 7,5 \times 5,3 \times 3$ modules \\
\hline \multirow{3}{*}{$\begin{array}{l}\text { Alignment } \\
\text { Pattern }\end{array}$} & \multirow{3}{*}{$1: 1: 1: 1: 1$} & 3 Co-centric squares \\
\hline & & Higher version 2 \\
\hline & & $5 \times 5,3 \times 3,1 \times 1$ modules \\
\hline
\end{tabular}

\section{OBJECTIVE}

The objective of this article is to represent an easy way to generate QR code into VB.NET. It is an upgrade to the last version of VB (version 6.0) that conforms to the .NET platform. It is a programming language that directly supported programmable graphical user interfaces using languagesupplied objects. Here the provided code will make possible for a general user to simply type text in a vb.net form window 
and he will get a QR Code image. He can also save this image in jpeg format to the specified location or disk on his personal computer.

\section{PREVIOUS WORK}

\subsection{QR Code Data Representation for Mobile Augmented Reality}

In this position paper, they introduced using QR code for MAR applications. Since QR code can self-contain much information, we aimed to use this capacity to include metadata for MAR application covering from code metadata, content metadata and tracking metadata. Yet there are many issues to resolve, such as how to distinguish normal QR code from ARenabled QR code. Also similar visual codes need to be compared in tracking aspect for better performance in MAR and we can extend our proposed idea to other visual codes as well [11].

\subsection{Generating SMS (Short Message Service) in the form of Quick Response Code (QR-code)}

This article shows how a QR-code can be generated from a simple SMS. Developers began trying to expand on the current amount of bars within the barcode and how their positioning resides to allow further data capacities. The need for smaller barcodes also was another defining factor in QRCodes development [12].

\subsection{QR Code Security}

In this paper they outlined the dangers of possible attacks utilizing manipulated QR Codes. Since QR Codes gain increasing popularity through their use for marketing purposes, we expect that this kind of attack will receive more and more attention by the hacking community in the future. In addition to phishing, a multitude of other attack methods, both against humans and automated systems, might be performed using QR codes. This especially holds true if proper input sanitization is not performed prior to processing the contained data [13].

\subsection{QR Codes in Education}

In this paper, they provided a holistic view of using QR codes in industry and in education. We demonstrated three broad areas to apply QR codes in school education. First, we must categorically prepare enough mobile devices for the activities. We definitely cannot expect students at the junior levels to carry the right mobile devices to schools. Second, despite it is quite easy to operate a mobile device equipped with a QR code reader, we noticed some students highly tilted the devices when they snapped the codes. Third, if the mobile devices are equipped with Wi-Fi access and the activities do have the wireless coverage, the cost for communication would be minimal. In this paper and there are many creative ideas waiting for us to explore. Also, this paper can be served as the first step for the readers to investigate this exciting topic of mobile learning [14].

\subsection{The Expectations of Quick Response (QR) Codes in Print Media: An Empirical Data Research Anthology}

QR Codes have only recently gained popularity in the United States commercial markets starting in 2010 and are still a relatively new tool as of early 2012. For this reason, little scholarly research has investigated awareness or the effectiveness of QR codes in a marketing sense. This study had to rely heavily on research and information collected from trade magazines, popular press mediums, past research studies conducted for different purposes, and information posted on professional social networking sites, such as LinkedIn[15].

\subsection{Research on Distortion Correction of QR Code Images}

In this paper, an algorithm for the distorted QR code image correction is presented. The gradient-based HT is modified when finding out the vertices of $\mathrm{QR}$ code symbol. It is achieved by 1 ) calculating the adaptive parameters $\mathrm{G}$ and $\mathrm{k}$ and getting the 8 longest lines (see Algorithm 1) and 2) finding out the four vertices of QR code symbol (see Algorithm 2). As a result, the $\mathrm{QR}$ code symbol can be corrected [16].

\section{METHODOLOGY}

As we describe in this article you must vb.net as well as OnBarcode.BarcodeGenerator.DotnetSuite. After this you can use the below code to generate QR Code through vb.net. You may set the properties of QR Code like data, ECL, size as per your requirement. You can also save this QR Code picture on your disk. the code for this is given below.

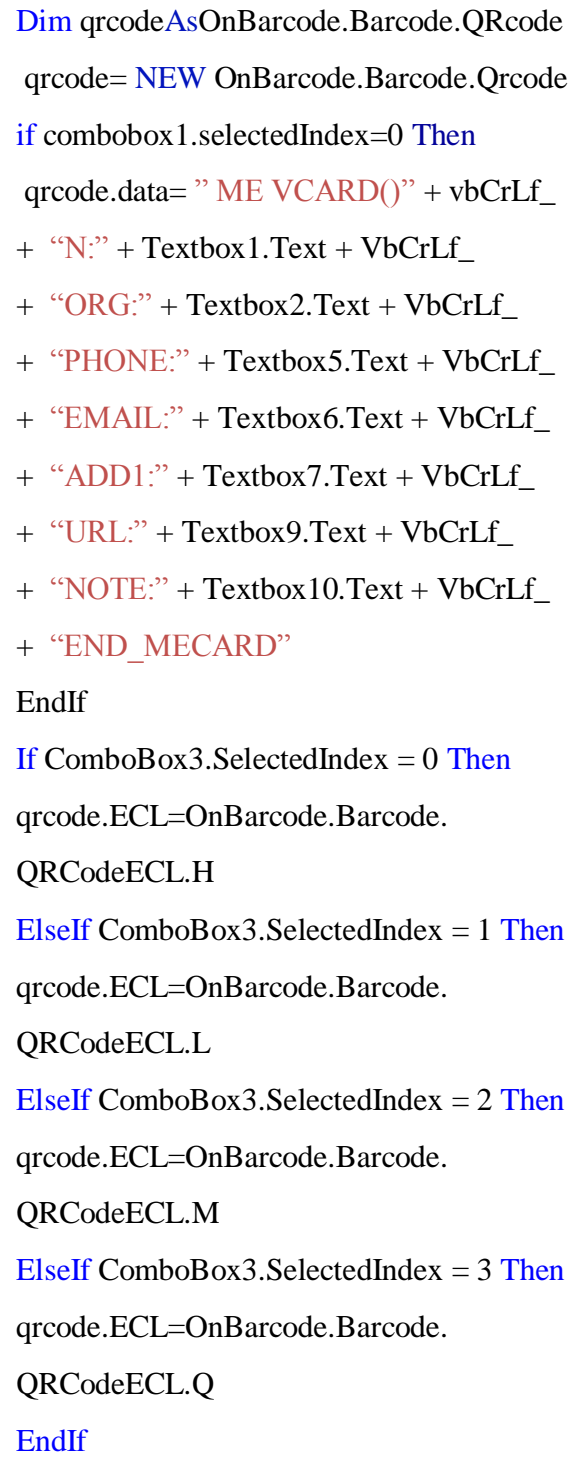

Dim qrcodeAsOnBarcode.Barcode.QRcode if combobox 1 . selectedIndex $=0$ Then

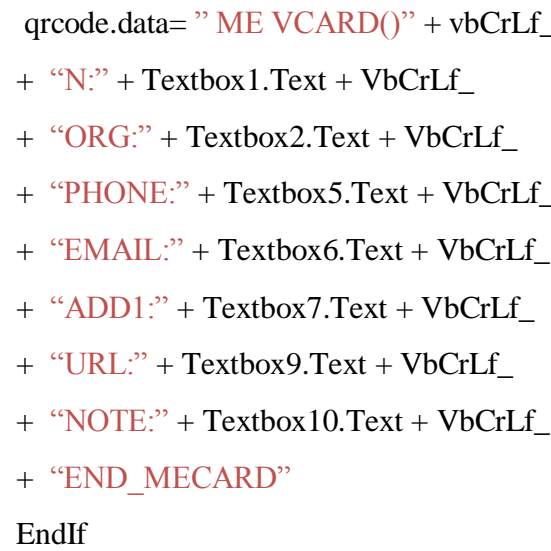

If ComboBox3.SelectedIndex $=0$ Then qrcode. $\mathrm{ECL}=\mathrm{OnB}$ arcode . Barcode.

QRCodeECL.H

ElseIf ComboBox3.SelectedIndex $=1$ Then 


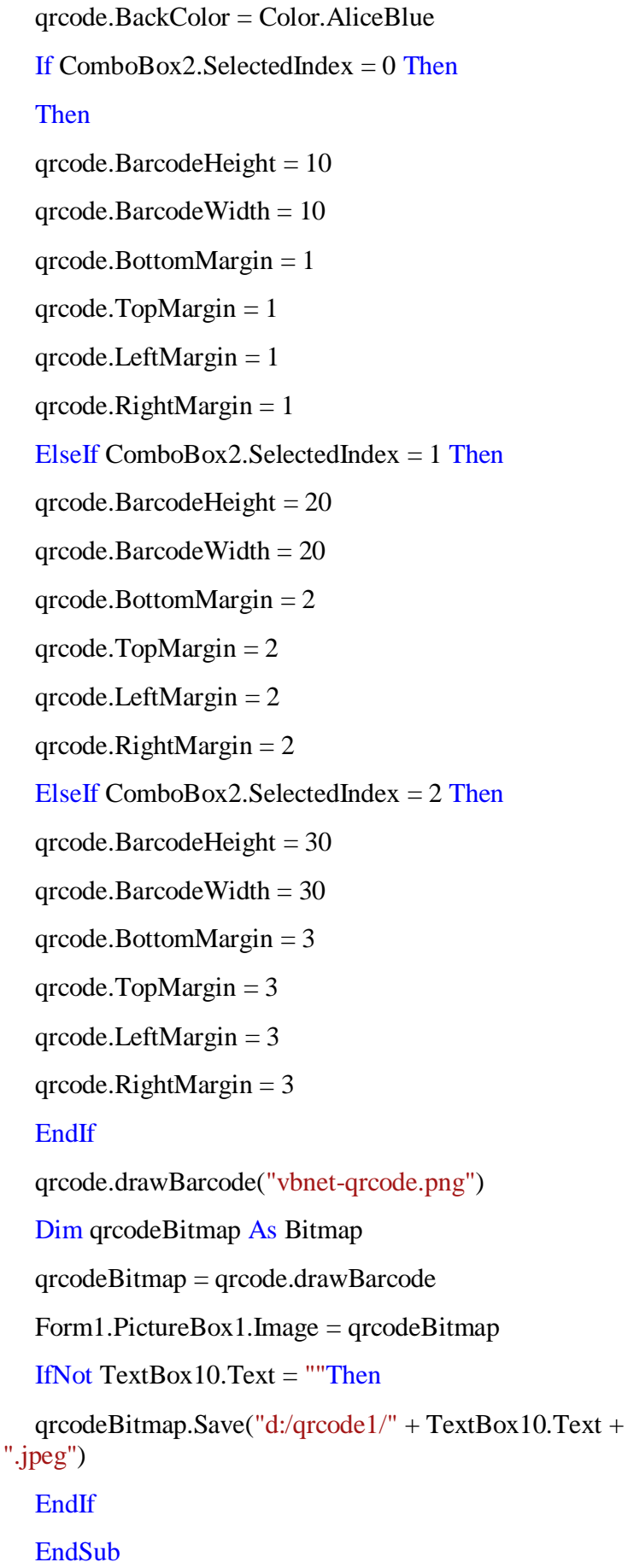

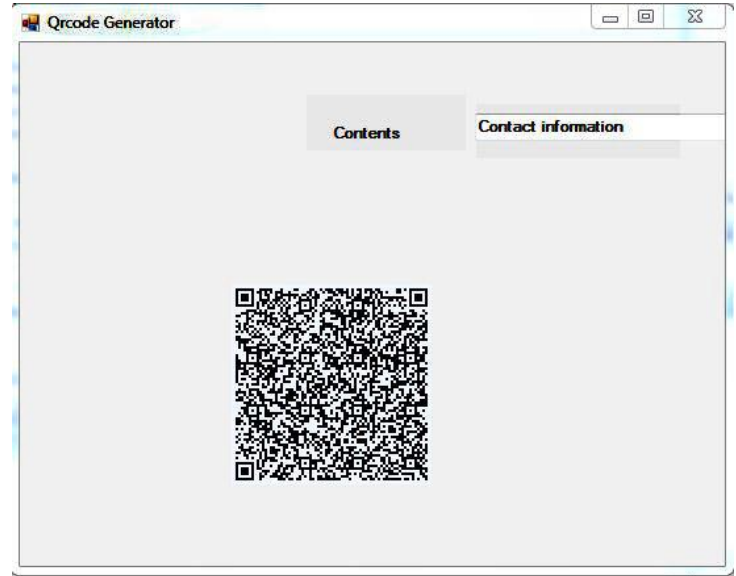

Figure 7: QR CodeGenerator

\section{CONCLUSION}

In this article we describe how to generate a $\mathrm{QR}$ code in VB.NET.A general user can generate his own QR code in a user friendly environment of vb.net and a programmer can generate software for this by following the steps given in this article.

\section{REFERENCES}

[1]. ISO/IEC 18004: ISO Standard on QR Code 2005 Bar Code Symbology Specification.

[2]. BorkoFurht(2011). Handbook of Augmented Reality.Springer.p. 341.

[3]. QR Code from Wikipedia, The free encyclopedia.

[4]. Section three QR Code, Tan Jin Soon, Executive Director, and Epcglobal Singapore Council.

[5]. QR Code.com. Denso-wave.com. Retrieved 23 April 2009.

[6]. ISO/IEC 18004: ISO Standard on QR Code 2005 Bar Code Symbology Specification.

[7]. JIS-X0510: Japan Industrial Standard.

[8]. Wakahara, Toshihiko; Yamamoto, Noriyasu; "Image Processing of 2-Dimensional Barcode", Conference on Network-Based Information Systems (NBiS), 2011 14th International.

[9]. Japanese Industrial Standards, "Two Dimensional Symbol-QR-Code-Basic Specification” JIS X 0510, October 2004.

[10]. T. J. Soo, “QR Code”, Synthesis Journal, pp..59-78 2008.

[11].http://webstaff.itn.liu.se/ perla/Siggraph2010/content/po sters/0148.pdf

http://www.ijcsmc.com/docs/papers/december2012/V1201212 03.pdf

[12].http://www.sba-research.org/wpcontent/uploads/publications/QR_Code_Security.pdf

[13].http://www.sicet.org/journals/jetde/jetde10/7-So.pdf

[14].http://www.uwlax.edu/urc/JURonline/PDF/2012/probst.ali.pdf

[15].http://www.ijcst.com/vol31/3/yunfei.pdf 